\title{
Characterization of quince (Cydonia oblonga Mill.) cultivars using SSR markers developed for apple
}

\author{
Halász, J. ${ }^{1}$, Hoffmann, V. ${ }^{1}$, Szabó, $Z_{.}^{2}$, Nyéki, J. ${ }^{2}$, Szabó, T. ${ }^{3}$ \& Hegedüs, A. ${ }^{1}$ \\ ${ }^{1}$ Corvinus University of Budapest, Faculty of Horticultural Science, Department of Genetics and Plant Breeding, \\ 1118 Budapest, 29 Villányi street, Hungary; \\ ${ }^{2}$ Institute for Extension and Development, University of Debrecen, 138 Böszörményi street, \\ H-4032 Debrecen, Hungary \\ ${ }^{3}$ Research and Extension Centre for Fruit Growing, 2 Vadastag street, H-4244 Újfehértó, Hungary
}

\begin{abstract}
Summary: Quince (Cydonia oblonga Mill.) is a minor fruit crop, which is primarily used for marmalade, jam and sauce. Very few quince cultivars are known all over the world and in many cases similar names are used for presumably different cultivars. The aim of the present study was to evaluate and characterize the genetic diversity of 36 quince cultivars and selections with SSR markers. Seven out of 8 SSR markers designed from apple sequences could successfully yield amplification also in quince cultivars. Number of alleles per locus ranged from 2 to 3 alleles. These allele numbers are quite low when compared to apple. It is supposed to be the consequence of a genetic bottleneck. In spite of the low allele number per locus, the 36 quince cultivars formed 30 different genotypes. The ratio of homozygosity was low, which might be coupled with the self(in)compatibility phenotype of quinces. SSR markers proved unable to differentiate putatively closely related cultivars (e.g. 'Bereczki' and 'Bereczki bőtermő'). In general, the level of polymorphism among the tested quince genotypes was much restricted due to the low allele number detected. However, it must be considered that the number of analysed SSR loci is not enough high to estimate the overall heterozygosity of the quince genome. Further experiments are needed and the SSR markers proved to be a reliable and useful tool for such analyses.
\end{abstract}

Key words: Cydonia oblonga, quince, genetic diversity, SSR

\section{Introduction}

The quince (Cydonia oblonga Mill.) belongs to the Rosaceae family, Maloideae subfamily, which includes commercially important fruits such as apples or pears. This subfamily comprises approximately 1000 species in 30 genera and is characterized by a distinctive fruit, pome, and a base chromosome number $(x)$ of 17 (Rodger \& Campbell, 2002). The quince was thought to have originated in Persia, Turkistan and the Caucasus. Its tree is used as rootstock for pear cultivars, while its fruit is used for making preserves, jam, marmalade, sauce and juice. In Hungary, it has been cultivated for a long time but its importance lags behind other fruit species. Sándor Brózik performed the domestication of foreign quince cultivars and collected native landraces and selections. The best cultivars grown in Hungary are 'Angersi', 'Bereczki bőtermő', 'Champion' and 'Mezőtúri' (Szabó, 1998). Only few breeders or researchers work with quince; that is why detailed descriptions of cultivars hardly exist. Characteristics of fruit and tree are so similar that it is very difficult to make a reliable classification. Differentiation of cultivars based on the shape of fruit: 'apple-shaped' (maliformis Mill.-Schneid.) and 'pear-shaped' (pyriformis Dierb.- F. Zinn) but the morphology of fruit could be influenced by the fruit set, the number of seeds and environmental factors (Hedrick, 1925). All over the world approximatley 40-50 cultivars are propagated, among them 10-15 are well-known (Szabó, 1998). The use of cultivar names is quite chaotic among different countries, for example the Romanian 'De Constantinapol' is distinct from the Hungarian 'Konstantinápolyi' (Bordeianu, 1964).

The DNA-based molecular markers have become increasingly popular in the characterization and identification of genetic resources because they are not influenced by the environmental factors and are more polymorphic. However, there is only one report on genetic study of quince cultivars using SSR markers. Yamamoto et al. (2004) characterized twenty quince cultivars from France, USA, Japan, Portugal and England using 77 SSR primers developed from the pear and apple genome. The development of SSR markers needs preliminary sequence information of the genome but it was noted that the use of heterologous SSR primers in closely related species was an effective approach because of the conserved flanking region of microsatellite repeats (Moore et al., 1991; Cipriani et al., 1999; Testolin et al., 2000; Dirlewanger et al., 2002). Liebhard et al. (2002) established that the tested SSR markers from apple was transferable and could make successful cross-amplification in other genera (Cotoneaster, Pyrus, Cydonia etc.) of the same Maloideae subfamily. In apple, SSR markers could have been used for the assessment of genetic diversity (Guarino et al., 2006; Pereira-Lorenzo et al., 
2007; Garkava-Gustavsson et al., 2008) and cultivar identification (Guilford et al., 1997; Galli et al., 2005).

The aim of the present study was to evaluate and characterize the genetic diversity of 36 quince cultivars and selections obtained from the valuable germplasm collection of Research and Extension Centre for Fruit Growing in Újehértó.

\section{Materials and methods}

Young leaves of 36 quince cultivars and selections were collected in the germplasm collection of the Research and Extension Centre for Fruit Growing, Úfehértó. Genomic DNA was extracted using a DNeasy Plant Mini Kit (Qiagen, Hilden, Germany). PCR was conducted using eight pairs of primers flanking SSR sequences: CH01f02, CH01h01 (Gianfranceschi et al., 1998), CH02c02a, CH02c09, CH03g04, CH04e03, CH05d11, CH05e03 (Liebhard et al., 2002). PCR was carried out in a PTC 200 thermocycler (MJ Research, Budapest, Hungary) using the program described for the primers. PCR was performed in $25 \mu$ l volume containing about $25 \mathrm{ng}$ of genomic DNA, $1 \mathrm{x}$ buffer, $2.5 \mathrm{mM}$ $\mathrm{MgCl}_{2}, 0.2 \mathrm{mM}$ dNTPs, $0.2 \mathrm{iM}$ each of primers and $1 \mathrm{U}$ Taq polymerase (Fermentas, Szeged, Hungary). To determine the exact size of different fragments, the PCR products amplified by fluorescently labelled forward primers (JOE or FAM) were run in an automated sequencer ABI PRISM 3100 Genetic Analyzer (Applied Biosystems, Budapest, Hungary). Band scoring was analyzed using ABI Genotyper 3.7 NT software.

\section{Results and Discussion}

Seven out of 8 SSR markers derived from apple genome could successfully produce amplified bands in quince cultivars. All these 7 primer pairs generated 1 or 2 discrete reproducible fragments. In cases when only one band was visible, its size is reported twice since the genotype is presumed to be homozygous. The allele sizes determined by the same markers were identical in all replications. Primer $\mathrm{CH} 05 \mathrm{e} 03$ could not amplify any fragment in the tested cultivars. Since no SSR markers have been developed in quince, it is considered that the use of transferable SSRs from apple could be suitable for the genetic identification of quince cultivars (Yamamoto et al., 2004). Number of alleles per locus ranged from 2 to 3 alleles (Figure 1): 3 alleles in $\mathrm{CH} 03 \mathrm{~g} 07$ locus (labelled as A1, A2, A3), 2 alleles in $\mathrm{CH} 04 \mathrm{e} 03$ locus (B1, B2), 3 alleles in CH05d11 locus (C1, C2, C3), 3 alleles in CH02C02 locus (D1, D2, D3), 3 alleles in $\mathrm{CH} 01 \mathrm{F0} 2$ locus (E1, E2, E3), 3 alleles in CH02C09 locus (F1, F2, F3) and 2 alleles in $\mathrm{CH} 01 \mathrm{H} 01$ locus (G1, G2). These allele numbers are quite low, the same loci in apple showed average of 9.2 allelic variants, from 6 to 13 alleles per locus (Liebhard et al., 2002; Galli et al., 2005). It is supposed to be the consequence of a genetic bottleneck, which might be at least partially the explanation why only a limited number of quince cultivars are known worldwide (Hedrick, 1925). Using RAPD analysis the tested cultivars showed a very similar banding pattern and it was impossible to differentiate them (Yamamoto et al., 2004). However, an isozyme analysis revealed a restricted genetic diversity but enabled to classify cultivars into several types (Sanchez et al., 1988).

In spite of the low allele number per locus, the 36 quince cultivars formed 30 different genotypes. From this point of view these SSR markers were effective for the characterization of quince cultivars with unknown origin. Yamamoto et al. (2004) found 12 different genotypes among 20 cultivars. The ratio of homozygosity was low, which seems to be very indicative considering that fruit set studies on the self-fertility properties of quince revealed many contradictions according to a wide range of environmental conditions (Szabó et al., 1999; Nyéki et al., 2003). If selfpollination is prevented by self-incompatibility, the level of heterozygosity is high as in case of apple (Guarino et al.,

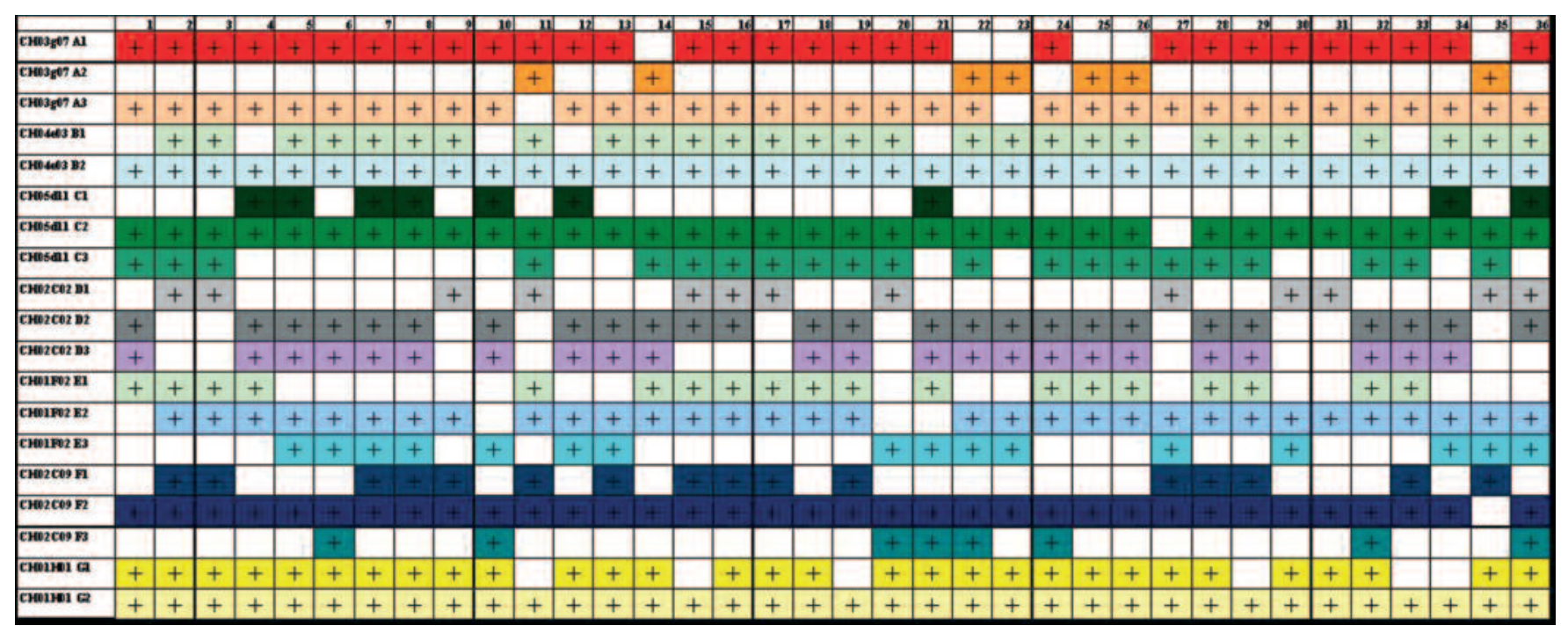

Figure 1: Frequency of alleles observed in 36 quince cultivars at each SSR locus. 
2006). As quince belongs to the Maloideae subfamily, a functional gametophytic self-incompatible system (GSI) can be presumed to control fruit set, nevertheless no data are available on molecular investigations (Halász, 2008). This would be, however, extremely important to provide more unequivocal results required for the clarification of the above described uncertainties. It must be mentioned that the number of analysed SSR loci is not enough to estimate the overall heterozygosity of the quince genome, further experiments are needed and the SSR markers proved to be a reliable and useful tool for such analyses.

Allelic compositions for each analysed quince cultivar are presented in Table 1. 'Bereczki' and 'Bereczki bőtermő' cultivars were characterized by the same genotype. It was

Table 1: Allelic compositions of 7 SSR loci for each analysed quince cultivar

\begin{tabular}{|c|c|}
\hline Cultivars & SSR genotypes \\
\hline 1. 162 & $\mathrm{~A}_{1} \mathrm{~A}_{3} \mathrm{~B}_{2} \mathrm{~B}_{2} \mathrm{C}_{2} \mathrm{C}_{3} \mathrm{D}_{2} \mathrm{D}_{3} \mathrm{E}_{1} \mathrm{E}_{1} \mathrm{~F}_{2} \mathrm{~F}_{2} \mathrm{G}_{1} \mathrm{G}_{2}$ \\
\hline 2. Almaalakú & $\mathrm{A}_{1} \mathrm{~A}_{3} \mathrm{~B}_{1} \mathrm{~B}_{2} \mathrm{C}_{2} \mathrm{C}_{3} \mathrm{D}_{1} \mathrm{D}_{1} \mathrm{E}_{1} \mathrm{E}_{2} \mathrm{~F}_{1} \mathrm{~F}_{2} \mathrm{G}_{1} \mathrm{G}_{2}$ \\
\hline 3. Almaalakú mezőtúri & $\mathrm{A}_{1} \mathrm{~A}_{3} \mathrm{~B}_{1} \mathrm{~B}_{2} \mathrm{C}_{2} \mathrm{C}_{3} \mathrm{D}_{1} \mathrm{D}_{1} \mathrm{E}_{1} \mathrm{E}_{2} \mathrm{~F}_{1} \mathrm{~F}_{2} \mathrm{G}_{1} \mathrm{G}_{2}$ \\
\hline 4. Angersi & $\mathrm{A}_{1} \mathrm{~A}_{3} \mathrm{~B}_{2} \mathrm{~B}_{2} \mathrm{C}_{1} \mathrm{C}_{2} \mathrm{D}_{2} \mathrm{D}_{3} \mathrm{E}_{1} \mathrm{E}_{2} \mathrm{~F}_{2} \mathrm{~F}_{2} \mathrm{G}_{1} \mathrm{G}_{2}$ \\
\hline 5. Aromate & $\mathrm{A}_{1} \mathrm{~A}_{3} \mathrm{~B}_{1} \mathrm{~B}_{2} \mathrm{C}_{1} \mathrm{C}_{2} \mathrm{D}_{2} \mathrm{D}_{3} \mathrm{E}_{2} \mathrm{E}_{3} \mathrm{~F}_{2} \mathrm{~F}_{2} \mathrm{G}_{1} \mathrm{G}_{2}$ \\
\hline 6. Auri & $\mathrm{A}_{1} \mathrm{~A}_{3} \mathrm{~B}_{1} \mathrm{~B}_{2} \mathrm{C}_{2} \mathrm{C}_{2} \mathrm{D}_{2} \mathrm{D}_{3} \mathrm{E}_{2} \mathrm{E}_{3} \mathrm{~F}_{2} \mathrm{~F}_{3} \mathrm{G}_{1} \mathrm{G}_{2}$ \\
\hline 7. Bereczki & $\mathrm{A}_{1} \mathrm{~A}_{3} \mathrm{~B}_{1} \mathrm{~B}_{2} \mathrm{C}_{1} \mathrm{C}_{2} \mathrm{D}_{2} \mathrm{D}_{3} \mathrm{E}_{2} \mathrm{E}_{3} \mathrm{~F}_{1} \mathrm{~F}_{2} \mathrm{G}_{1} \mathrm{G}_{2}$ \\
\hline 8. Bereczki bőtermő & $\mathrm{A}_{1} \mathrm{~A}_{3} \mathrm{~B}_{1} \mathrm{~B}_{2} \mathrm{C}_{1} \mathrm{C}_{2} \mathrm{D}_{2} \mathrm{D}_{3} \mathrm{E}_{2} \mathrm{E}_{3} \mathrm{~F}_{1} \mathrm{~F}_{2} \mathrm{G}_{1} \mathrm{G}_{2}$ \\
\hline 9. Bicskei óriás & $\mathrm{A}_{1} \mathrm{~A}_{3} \mathrm{~B}_{1} \mathrm{~B}_{2} \mathrm{C}_{2} \mathrm{C}_{2} \mathrm{D}_{1} \mathrm{D}_{1} \mathrm{E}_{2} \mathrm{E}_{2} \mathrm{~F}_{1} \mathrm{~F}_{2} \mathrm{G}_{1} \mathrm{G}_{2}$ \\
\hline 10. Champion & $\mathrm{A}_{1} \mathrm{~A}_{3} \mathrm{~B}_{2} \mathrm{~B}_{2} \mathrm{C}_{1} \mathrm{C}_{2} \mathrm{D}_{2} \mathrm{D}_{3} \mathrm{E}_{3} \mathrm{E}_{3} \mathrm{~F}_{2} \mathrm{~F}_{3} \mathrm{G}_{1} \mathrm{G}_{2}$ \\
\hline 11. Cserszegi birs & $\mathrm{A}_{1} \mathrm{~A}_{2} \mathrm{~B}_{1} \mathrm{~B}_{2} \mathrm{C}_{2} \mathrm{C}_{3} \mathrm{D}_{1} \mathrm{D}_{1} \mathrm{E}_{1} \mathrm{E}_{2} \mathrm{~F}_{1} \mathrm{~F}_{2} \mathrm{G}_{2} \mathrm{G}_{2}$ \\
\hline 12. De Husi & $\mathrm{A}_{1} \mathrm{~A}_{3} \mathrm{~B}_{2} \mathrm{~B}_{2} \mathrm{C}_{1} \mathrm{C}_{2} \mathrm{D}_{2} \mathrm{D}_{3} \mathrm{E}_{2} \mathrm{E}_{3} \mathrm{~F}_{2} \mathrm{~F}_{2} \mathrm{G}_{1} \mathrm{G}_{2}$ \\
\hline 13. Deák Csokonai & $\mathrm{A}_{1} \mathrm{~A}_{3} \mathrm{~B}_{1} \mathrm{~B}_{2} \mathrm{C}_{2} \mathrm{C}_{2} \mathrm{D}_{2} \mathrm{D}_{3} \mathrm{E}_{2} \mathrm{E}_{3} \mathrm{~F}_{1} \mathrm{~F}_{2} \mathrm{G}_{1} \mathrm{G}_{2}$ \\
\hline 14. Dunaföldvári & $\mathrm{A}_{2} \mathrm{~A}_{3} \mathrm{~B}_{1} \mathrm{~B}_{2} \mathrm{C}_{2} \mathrm{C}_{3} \mathrm{D}_{2} \mathrm{D}_{3} \mathrm{E}_{1} \mathrm{E}_{2} \mathrm{~F}_{2} \mathrm{~F}_{2} \mathrm{G}_{1} \mathrm{G}_{2}$ \\
\hline 15. Fehérvári & $\mathrm{A}_{1} \mathrm{~A}_{3} \mathrm{~B}_{1} \mathrm{~B}_{2} \mathrm{C}_{2} \mathrm{C}_{3} \mathrm{D}_{1} \mathrm{D}_{2} \mathrm{E}_{1} \mathrm{E}_{2} \mathrm{~F}_{1} \mathrm{~F}_{2} \mathrm{G}_{2} \mathrm{G}_{2}$ \\
\hline 16. Fuji & $\mathrm{A}_{1} \mathrm{~A}_{3} \mathrm{~B}_{1} \mathrm{~B}_{2} \mathrm{C}_{2} \mathrm{C}_{3} \mathrm{D}_{1} \mathrm{D}_{2} \mathrm{E}_{1} \mathrm{E}_{2} \mathrm{~F}_{1} \mathrm{~F}_{2} \mathrm{G}_{1} \mathrm{G}_{2}$ \\
\hline 17. Györkönyi almaalakú & $\mathrm{A}_{1} \mathrm{~A}_{3} \mathrm{~B}_{1} \mathrm{~B}_{2} \mathrm{C}_{2} \mathrm{C}_{3} \mathrm{D}_{1} \mathrm{D}_{1} \mathrm{E}_{1} \mathrm{E}_{2} \mathrm{~F}_{1} \mathrm{~F}_{2} \mathrm{G}_{1} \mathrm{G}_{2}$ \\
\hline 18. Konstantinápolyi & $\mathrm{A}_{1} \mathrm{~A}_{3} \mathrm{~B}_{1} \mathrm{~B}_{2} \mathrm{C}_{2} \mathrm{C}_{3} \mathrm{D}_{2} \mathrm{D}_{3} \mathrm{E}_{1} \mathrm{E}_{2} \mathrm{~F}_{2} \mathrm{~F}_{2} \mathrm{G}_{1} \mathrm{G}_{2}$ \\
\hline 19. Lengyel & $\mathrm{A}_{1} \mathrm{~A}_{3} \mathrm{~B}_{1} \mathrm{~B}_{2} \mathrm{C}_{2} \mathrm{C}_{3} \mathrm{D}_{2} \mathrm{D}_{3} \mathrm{E}_{1} \mathrm{E}_{3} \mathrm{~F}_{1} \mathrm{~F}_{2} \mathrm{G}_{2} \mathrm{G}_{2}$ \\
\hline 20. Leskovacka & $\mathrm{A}_{1} \mathrm{~A}_{3} \mathrm{~B}_{2} \mathrm{~B}_{2} \mathrm{C}_{1} \mathrm{C}_{2} \mathrm{D}_{1} \mathrm{D}_{1} \mathrm{E}_{3} \mathrm{E}_{3} \mathrm{~F}_{2} \mathrm{~F}_{3} \mathrm{G}_{1} \mathrm{G}_{2}$ \\
\hline 21. Mezőkövesdi & $\mathrm{A}_{1} \mathrm{~A}_{3} \mathrm{~B}_{1} \mathrm{~B}_{1} \mathrm{C}_{1} \mathrm{C}_{2} \mathrm{D}_{2} \mathrm{D}_{3} \mathrm{E}_{1} \mathrm{E}_{3} \mathrm{~F}_{2} \mathrm{~F}_{3} \mathrm{G}_{1} \mathrm{G}_{2}$ \\
\hline 22. Mezőtúri & $\mathrm{A}_{2} \mathrm{~A}_{3} \mathrm{~B}_{1} \mathrm{~B}_{2} \mathrm{C}_{2} \mathrm{C}_{3} \mathrm{D}_{2} \mathrm{D}_{3} \mathrm{E}_{2} \mathrm{E}_{3} \mathrm{~F}_{2} \mathrm{~F}_{3} \mathrm{G}_{1} \mathrm{G}_{2}$ \\
\hline 23. Moldova nesti & $\mathrm{A}_{2} \mathrm{~A}_{2} \mathrm{~B}_{1} \mathrm{~B}_{2} \mathrm{C}_{2} \mathrm{C}_{2} \mathrm{D}_{2} \mathrm{D}_{3} \mathrm{E}_{2} \mathrm{E}_{3} \mathrm{~F}_{2} \mathrm{~F}_{2} \mathrm{G}_{1} \mathrm{G}_{2}$ \\
\hline 24. Nyakas birs & $\mathrm{A}_{1} \mathrm{~A}_{3} \mathrm{~B}_{1} \mathrm{~B}_{2} \mathrm{C}_{2} \mathrm{C}_{3} \mathrm{D}_{2} \mathrm{D}_{3} \mathrm{E}_{1} \mathrm{E}_{2} \mathrm{~F}_{2} \mathrm{~F}_{3} \mathrm{G}_{1} \mathrm{G}_{2}$ \\
\hline 25. Olasz 3 & $\mathrm{~A}_{2} \mathrm{~A}_{3} \mathrm{~B}_{1} \mathrm{~B}_{2} \mathrm{C}_{2} \mathrm{C}_{3} \mathrm{D}_{2} \mathrm{D}_{3} \mathrm{E}_{1} \mathrm{E}_{2} \mathrm{~F}_{2} \mathrm{~F}_{2} \mathrm{G}_{1} \mathrm{G}_{2}$ \\
\hline 26. Óriás birs & $\mathrm{A}_{2} \mathrm{~A}_{3} \mathrm{~B}_{1} \mathrm{~B}_{2} \mathrm{C}_{2} \mathrm{C}_{3} \mathrm{D}_{2} \mathrm{D}_{3} \mathrm{E}_{1} \mathrm{E}_{2} \mathrm{~F}_{2} \mathrm{~F}_{2} \mathrm{G}_{1} \mathrm{G}_{2}$ \\
\hline 27. Örsi birs & $\mathrm{A}_{1} \mathrm{~A}_{3} \mathrm{~B}_{2} \mathrm{~B}_{2} \mathrm{C}_{3} \mathrm{C}_{3} \mathrm{D}_{1} \mathrm{D}_{1} \mathrm{E}_{2} \mathrm{E}_{3} \mathrm{~F}_{1} \mathrm{~F}_{2} \mathrm{G}_{1} \mathrm{G}_{2}$ \\
\hline 28. Patyi almaalakú & $\mathrm{A}_{1} \mathrm{~A}_{3} \mathrm{~B}_{1} \mathrm{~B}_{2} \mathrm{C}_{2} \mathrm{C}_{3} \mathrm{D}_{2} \mathrm{D}_{3} \mathrm{E}_{1} \mathrm{E}_{2} \mathrm{~F}_{1} \mathrm{~F}_{2} \mathrm{G}_{1} \mathrm{G}_{2}$ \\
\hline 29. Pomázi & $\mathrm{A}_{1} \mathrm{~A}_{3} \mathrm{~B}_{1} \mathrm{~B}_{2} \mathrm{C}_{2} \mathrm{C}_{3} \mathrm{D}_{2} \mathrm{D}_{3} \mathrm{E}_{1} \mathrm{E}_{2} \mathrm{~F}_{1} \mathrm{~F}_{2} \mathrm{G}_{2} \mathrm{G}_{2}$ \\
\hline 30. Radónia & $\mathrm{A}_{1} \mathrm{~A}_{3} \mathrm{~B}_{1} \mathrm{~B}_{2} \mathrm{C}_{2} \mathrm{C}_{2} \mathrm{D}_{1} \mathrm{D}_{1} \mathrm{E}_{2} \mathrm{EeF}_{2} \mathrm{~F}_{2} \mathrm{G}_{1} \mathrm{G}_{2}$ \\
\hline 31. Román portugál & $\mathrm{A}_{1} \mathrm{~A}_{3} \mathrm{~B}_{2} \mathrm{~B}_{2} \mathrm{C}_{2} \mathrm{C}_{2} \mathrm{D}_{1} \mathrm{D}_{1} \mathrm{E}_{2} \mathrm{E}_{2} \mathrm{~F}_{2} \mathrm{~F}_{2} \mathrm{G}_{1} \mathrm{G}_{2}$ \\
\hline 32. Szentlörinci & $\mathrm{A}_{1} \mathrm{~A}_{3} \mathrm{~B}_{1} \mathrm{~B}_{2} \mathrm{C}_{2} \mathrm{C}_{3} \mathrm{D}_{2} \mathrm{D}_{3} \mathrm{E}_{1} \mathrm{E}_{2} \mathrm{~F}_{2} \mathrm{~F}_{3} \mathrm{G}_{1} \mathrm{G}_{2}$ \\
\hline 33. Trimoacium & $\mathrm{A}_{1} \mathrm{~A}_{3} \mathrm{~B}_{2} \mathrm{~B}_{2} \mathrm{C}_{2} \mathrm{C}_{3} \mathrm{D}_{2} \mathrm{D}_{3} \mathrm{E}_{1} \mathrm{E}_{2} \mathrm{~F}_{1} \mathrm{~F}_{2} \mathrm{G}_{2} \mathrm{G}_{2}$ \\
\hline 34. Triumph & $\mathrm{A}_{1} \mathrm{~A}_{3} \mathrm{~B}_{1} \mathrm{~B}_{2} \mathrm{C}_{1} \mathrm{C}_{2} \mathrm{D}_{2} \mathrm{D}_{3} \mathrm{E}_{1} \mathrm{E}_{2} \mathrm{~F}_{2} \mathrm{~F}_{2} \mathrm{G}_{2} \mathrm{G}_{2}$ \\
\hline 35. Váli birs & $\mathrm{A}_{2} \mathrm{~A}_{3} \mathrm{~B}_{1} \mathrm{~B}_{2} \mathrm{C}_{2} \mathrm{C}_{3} \mathrm{D}_{1} \mathrm{D}_{1} \mathrm{E}_{2} \mathrm{E}_{3} \mathrm{~F}_{1} \mathrm{~F}_{1} \mathrm{G}_{1} \mathrm{G}_{2}$ \\
\hline 36. Vranja & $\mathrm{A}_{1} \mathrm{~A}_{3} \mathrm{~B}_{1} \mathrm{~B}_{2} \mathrm{C}_{1} \mathrm{C}_{2} \mathrm{D}_{1} \mathrm{D}_{2} \mathrm{E}_{2} \mathrm{E}_{3} \mathrm{~F}_{2} \mathrm{~F}_{3} \mathrm{G}_{1} \mathrm{G}_{2}$ \\
\hline
\end{tabular}

expectable because 'Bereczki bőtermő' can be supposed to be a close relative of 'Bereczki'. Generally, sport mutants are indistinguishable from their progenitor genotype since their genomes differ only in a small portion, which influences a quantitative trait, for example yield or productivity. The chance to find this region by using SSR markers is very small. 'Györkönyi almaalakú', 'Almaalakú' and 'Almaalakú mezötúri' carried the same SSR-alleles. Their fruits are very similar in morphology but the fruit shape is not the best feature to differentiate cultivars since it may vary year by year (Hedrick, 1925; Szabó, 1998). To confirm this, the cultivar 'Patyi almaalakú' was characterized by a different genotype. SSR-genotypes of 'Dunaföldvári', 'Olasz 3' and 'Óriás birs' were identical. Two other cultivars, 'Szentlörinci' and 'Nyakas birs' also possessed the same allelic composition. All other cultivars were characterized by different genotypes; however, it must be added that most of them differed only in one or two loci. The level of polymorphism was much restricted due to the low allele number detected. Still quince remains to be a cryptic species within the Maloideae subfamily, requiring more detailed analyses for a satisfactory characterization.

\section{Acknowledgements}

This work was financed by the NKTH-OTKA K68921. Júlia Halász and Attila Hegedüs are grateful for receiving a János Bolyai Scholarship, HAS, and Attila Hegedüs for the prize Excellence in science, Corvinus University of Budapest.

\section{References}

Bordeianu, T. (1964): Pomologia Republicii Populare Romine III. Parul -Gutuiul-Mosmonul-Scopusul. Editura Akademii Republicii Popularse Romine, Bucuresti.

Cipriani G., Lot G., Huang W. G., Marrazzo M. T., Peterlunger E. \& Testolin R. (1999): AC/GT and AG/CT microsatellite repeats in peach (Prunus persica (L) Batsch) : Isolation, characterisation and cross-species amplification in Prunus. Theoretical and Applied Genetics, 99: 65-72.

Dirlewanger, E., Cosson, P., Tavaud, M., Aranzana, M.J., Poizat, C., Zanetto, A., Arús, P. \& Laigret, F. (2002): Development of microsatellite markers in peach [Prunus persica (L.) Batsch] and their use in genetic diversity analysis in peach and sweet cherry (Prunus avium L.). Theoretical and Applied Genetics, 105: 127-138.

Galli, Zs., Halász, G., Kiss, E., Heszky, L. \& Dobránszki, J. (2005): Molecular identification of commercial apple cultivars with microsatellite markers. HortScience, 40 (7): 1974-1977.

Garkava-Gustavsson, L., Kolodinska Brantestam, A., Sehic, J. \& Nybom, H. (2008): Molecular characterisation of indigenous Swedish apple cultivars based on SSR and S-allele analysis. Hereditas, 1-14.

Gianfranceschi, L., Seglias, N., Tarchini, R., Komjanc, M. \& Gessler, C. (1998): Simple sequence repeats for the genetic analysis of apple. Theor Appl Genet, 96: 1069-1076. 
Guarino, C., Santoro, S., De Simone, L., Lain, O., Cipriani, G. \& Testolin, R. (2006): Genetic diversity in a collection of ancient cultivars of apple (Malus $x$ domestica Borkh.) as revealed by SSRbased fingerprinting. Journal of Horticultural Science \& Biotechnology, 81 (1): 39-44.

Guilford, P., Prakash, S., Zhu, J. M., Rikkerink, E., Gardiner, S., Bassett, H. \& Foster, R. (1997): Microsatellites in Malus $x$ domestica (apple): abundance, polymorphism and cultivar identification. Theor Appl Genet, 94: 249-254.

Halász, J. (2008): Chapter 9.2.3. Other pomaceous species. In: Morphology, Biology and Fertility of Flowers in Temperate Zone Fruits (Eds.: Nyéki, J., Soltész, M., Szabó, Z.). Akadémiai Kiadó, Budapest, pp. 258-259.

Hedrick, U.P. (1925): Systematic Pomology. The Macmillan Company, New York.

Liebhard, R., Gianfranceschi, L., Koller, B., Ryder, C. D., Tarchini, R., Van De Weg, E. \& Gessler, C. (2002): Development and characterisation of 140 microsatellites in apple (Malus $x$ domestica Borkh.). Molecular Breeding, 10: 217-241.

Moore, S.S., Sargent, L.L., King, T.J., Mattick, J.S., Georges, M. \& Hetzel, D.J.S. (1991): The conservation of dinucleotide microsatellites among mammalian genomes allows the use of heterologous PCR primer pairs in closly related species. Genomics, 10: 654-660.

Nyéki, J., Soltész, M. \& Szabó, Z. (2003): Quince. In: Kozma, P., Nyéki, J., Soltész, M., Szabó, Z. (Eds.): Floral Biology, Pollination and Fertilisation in Temperate Zone Fruit Species and Grape. pp. 333-339. Akadémiai Kiadó, Budapest.
Pereira-Lorenzo, S, Ramos-Cabrer, A.M. \& Diáz-Hernández, M.B. (2007): Evaluation of genetic identity and variation of local apple cultivars (Malus $x$ domestica Borkh.) from Spain using microsatellite markers. Genet. Res. Crop. Evol. 54: 405-420.

Rodger, C.E. \& Campbell, C.S. (2002): The origin of the apple subfamily (Maloideae; Rosaceae) is clarified by DNA sequence data from duplicated GBSSI genes. American Journal of Botany, 89 (9): 1478-1484.

Sanchez, E.E., Menendez, R. A., Daley, L.S. Boone, R.B., Jahn, O.L. \& Lombard, P.B. (1988): Characterization of quince (Cydonia) cultivars using polyacrylamide gel electrophoresis. J. Environ. Hort., 6: 53-59.

Szabó, T. (1998): Birs. In: Gyümölcsfajta-ismeret és használat (Ed.:Soltész, M.). Mezőgazda Kiadó, Budapest, pp. 187-195.

Szabó, T., Nyéki, J., Soltész, M., Szabó, Z. \& Tóth, T. (1999): Time of flowering and fertilisation of quince varieties. International Journal of Horticultural Science, 5: 9-15.

Testolin, R., Marrazzo, T., Cipriani, G., Quarta, R., Verde, I., Dettori, T., Pancaldi, M. \& Sansavini, S. (2000): Microsatellite DNA in peach (Prunus persica (L.) Batsch) and its use in fingerprinting and testing the genetic origin of cultivars. Genome, 43: 512-520.

Yamamoto, T., Kimura, T., Soejima, J., Ban, Y. \& Hayashi, T. (2004): Identification of quince varieties using SSR markers developed from pear and apple. Breeding Science, 54: 239-244. 